\title{
The effect of interfaces in liquid phase electron microscopy from an empirical viewpoint
}

Patricia Abellan ${ }^{1}$ and Jay LaVerne ${ }^{2}$

${ }^{1}$ IMN / University of Nantes, Nantes, France, ${ }^{2}$ Radiation Lab. and Dep. of Phys. / University of Notre Dame, Notre Dame, Indiana, United States

Nanoscale processes in liquids can now be studied in real time via the combination of liquid-cells and electron microscopes, EMs, (also termed liquid-cell EM, LCEM) as well as using dedicated environmental EMs. A major point of concern with this technology is the damage of samples in the electron beam due to radiolysis, which limits what can be measured and correctly interpreted in LCEM. The radiolysis of molecules by the effect of high energy electrons is unavoidable and finding means to perform experiments while keeping the lowest total dose and dose rate possible is a main focus in the field. For many experiments and specimens, however, current standards are still insufficient. [1]

Radiation chemistry involving ionizing sources initially produces localized regions of medium cations and low-energy free electrons.[2] The low dielectric constants of organic media lead to fast neutralization reactions and the production of the complete manifold of excited states as the energy cascades down from the ionization continuum. Low-energy electrons are an integral part of the resulting radiation chemical process. Cooling the specimen to liquid nitrogen temperatures, $77 \mathrm{~K}$, has been one of the key ideas behind the success of cryogenic electron microscopy, cryo-EM, since it minimizes the spread of damage to nearby areas by reducing the mobility of molecular and radical species. However, while cryogenic working conditions preserve the structure of biological molecules to higher electron fluences and thus images of undamaged biomolecules can be formed with larger signals, electronic transitions are temperature independent. The use of cryogenic temperatures, therefore, doesn't prevent the production of molecular excited states nor the mobility of secondary electrons, which are still able to move efficiently at $77 \mathrm{~K}$.

In LCEM two different strategies, beyond minimization of electron dose or optimization of signal detection during experiments, have been proposed. These strategies have been mostly adapted from existing knowledge from radiation chemistry to their application in the electron microscope: the use of modelling to test the effects of individual experimental parameter changes in the experiments [3] and methods for intercepting radicals [4]. Both of these strategies would benefit from a better understanding (or rather a direct measurement) of the effects of radiation chemistry in the electron microscope. In this presentation, I will present relevant aspects on the decomposition of molecules, with a special focus on the effect of interfaces. The possibility of using the EM as an efficient tool to probe specific radiation chemical pathways will be discussed. [5] If time allows, a calibration methodology for the radiation chemistry of aqueous solutions will be introduced.

\section{ACKNOWLEDGEMENTS:}

PA acknowledges the financial support by the NExT initiative through national funding by the French National Research Agency (ANR) under the Programme d'investissements d'Avenir (with reference ANR16-IDEX-0007). The e-BRIDGE project also receives financial support from the Pays de la Loire Region and Nantes Métropole. JAL acknowledges the financial support of the Division of Chemical Sciences, Geosciences and Biosciences, Basics Energy Sciences, Office of Science, U.S. DOE through grant number DE-FC02-04ER15533 


\section{References}

[1] de Jonge and Peckys, ACS Nano 10 (2016) 9061-9063; T. H. Moser et al. Micron 117 (2019), 8-15

[2] LaVerne, J.A., 2004. Radiation Chemical Effects of Heavy Ions, in: Mozumder, A., Hatano, Y. (Eds.), Charged Particle and Photon Interactions with Matter. Marcel Dekker, Inc, New York, pp. 403-429.

[3] N. M. Schneider et al., The Journal of Phys. Chem. C (2014) 118, 22373-22382; J.H. Park et al., Nano letters 15 (2015), 5314-5320; Gupta et al., Nanoscale (2018) 10, 7702-7710

[4] Woehl \& Abellan, Journal of Microsc. (2017) 265, 135-147; Sutter et al., Nat. Comm. (2014) 5, 1-9

[5] P. Abellan, J. LaVerne et al. In preparation 